\title{
Carleman Estimate for a Singulary Perturbed Degenerated Parabolic Equation
}

\author{
Zeine Sid Elemine ${ }^{1}$, Ibrahima Faye ${ }^{2}$, Alassane $\mathrm{Sy}^{2}$, Diaraf Seck ${ }^{3}$ \\ ${ }^{1}$ Department of Mathematics, Faculty of Sciences and Technologies, Cheikh Anta Diop University, Dakar, Senegal \\ ${ }^{2}$ Department of Mathematics, Faculty of Applied Sciences and Communications Technologies, Alioune Diop University, Bambey, Senegal \\ ${ }^{3}$ Department of Mathematics of Decision, Faculty of Economics and Management, Cheikh Anta Diop University, Dakar, Senegal
}

Email address:

zeine1977@hotmail.com (Z. S. Elemine), ibrahima.faye@uadb.edu.sn (I. Faye), alassane.sy@uadb.edu.sn (Alassane Sy)

\section{To cite this article:}

Zeine Sid Elemine, Ibrahima Faye, Alassane Sy, Diaraf Seck. Carleman Estimate for a Singulary Perturbed Degenerated Parabolic Equation. Science Journal of Applied Mathematics and Statistics. Vol. 9, No. 5, 2021, pp. 113-125. doi: 10.11648/j.sjams.20210905.11

Received: July 23, 2021; Accepted: August 18, 2021; Published: October 29, 2021

\begin{abstract}
In this paper, we are concerned with the the internal control of an elliptic singularly perturbed degenerated parabolic equation. This parabolic equation models sand transport problem near the coast in areas subjected to the tide. We study first the null controllability result of the parabolic equation modeling sand transport equation. The limit problem obtained by homogenization problem is also considered. We use distributed and bounded controls supported on a small open set of the initial domain. We prove the null controllability of the system at any time by using observability inequality for both problem. For this purpose, a specific carleman estimate for the solutions of degenerate adjoint limit problem is also proved.
\end{abstract}

Keywords: PDE Parabolic, Null Controllability, Sand Transport Model, Carlman Estimates, Observability Inequality

\section{Introduction and Results}

This paper, we focus on the study of null controllability of a singularly perturbed and degenerated parabolic partial differential equation. This kind of PDE arises from the modeling sand transport on the seabed in short time. The model is described in I. Faye and al. [9]. In the case of linear systems, exact controllability or null controllability is a

$$
\left\{\begin{array}{c}
\frac{\partial z^{\epsilon}}{\partial t}-\frac{1}{\epsilon} \nabla \cdot\left(\mathcal{A}^{\epsilon} \nabla z^{\epsilon}\right) \\
z^{\epsilon}(x, 0) \stackrel{\stackrel{=}{=} z_{0}(x) \text { in } \mathbb{T}^{2}}{ }
\end{array}\right.
$$

Where $\mathcal{A}^{\epsilon}$ and $\mathcal{C}^{\epsilon}$ are regular coefficients and $z_{0} \in L^{2}\left(\mathbb{T}^{2}\right)$. The function $z^{\epsilon}=z^{\epsilon}(t, x)$, is the dimensionless seabed altitude at $t$ and in $x$. For a given constant $T, t \in[0, T)$, stands for the dimensionless time and $x=\left(x_{1}, x_{2}\right) \in \mathbb{T}^{2}, \mathbb{T}^{2}$ being the two dimensional torus $\mathbb{R}^{2} / \mathbb{Z}^{2}$, is the dimensionless position variable.

Existence and uniqueness of solutions to (1) has been studied in [9] in the framework of periodic solutions if $\mathcal{A}^{\epsilon}$ and $\mathcal{C}^{\epsilon}$ and regular and bounded functions and can be set in widely study by many authors and for various methods see for example Russell [15], Lebeau-Robbiano [12], J. L. Lions [13]. Many other results of the null controlability of heat equation can also be found in G. Lebeau and L. Robbiano [12] and A. V. Fursikov and O. Yu. Imanuvilov [11] and Dubova et al. $[6,7,8]$.

We are particularly interested on models on the general form of:

$=\frac{1}{\epsilon} \nabla \cdot \mathcal{C}^{\epsilon}$ in $(0, T) \times \mathbb{T}^{2}$

the form $\mathcal{A}^{\epsilon}(t, x)=\widetilde{\mathcal{A}}_{\epsilon}\left(t, \frac{t}{\epsilon}, x\right)$ and $\mathcal{C}^{\epsilon}(t, x)=\widetilde{\mathcal{C}}_{\epsilon}\left(t, \frac{t}{\epsilon}, x\right)$, where $\theta \rightarrow \widetilde{\mathcal{A}}_{\epsilon}(t, \theta, x), \widetilde{\mathcal{C}}_{\epsilon}\left(t, \frac{\theta}{x}\right)$ are 1 - periodic in $\theta$. We have also to recall that the coefficients $\mathcal{A}^{\epsilon}$ and $\mathcal{C}^{\epsilon}$ can vanish and if $\epsilon$ is too small, the diffusion coefficient tends to infinity. The solution $z^{\epsilon}$ to (1) is bounded in this space and belongs to $L^{\infty}\left([0, T), L^{2}\left(\mathbb{T}^{2}\right)\right)$.

Using a result due to Nguetseng [16], [1] and [10], Faye et al in [9], proved that $z^{\epsilon}$ solution to (1) two-scale converges to a profile $U \in L^{\infty}\left([0, T), L_{\#}^{\infty}\left(\mathbb{R}, L^{2}\left(\mathbb{T}^{2}\right)\right)\right)$ solution to 


$$
\left.\frac{\partial U}{\partial \theta}-\nabla \cdot(\widetilde{\mathcal{A}}(t, . . .) \nabla U)=\nabla \cdot \widetilde{\mathcal{C}}(t, ., .) \text { in }\right] 0, T\left[\times \mathbb{R} \times \mathbb{T}^{2}\right.
$$

where $\widetilde{\mathcal{A}} \in L^{\infty}\left([0, T], L_{\#}^{\infty}\left(\mathbb{R}, L^{2}\left(\mathbb{T}^{2}\right)\right)\right)$ and $\widetilde{\mathcal{C}} \in\left(L^{\infty}\left([0, T], L_{\#}^{\infty}\left(\mathbb{R}, L^{2}\left(\mathbb{T}^{2}\right)\right)\right)\right)^{2}$ are respectively the two-scale limits of $\mathcal{A}^{\epsilon}$ and $\mathcal{C}^{\epsilon}$.

In this paper, we consider the following control problem: let $\omega$ be an open subset of $\mathbb{T}^{2}$ and $z^{\epsilon}$ the solution to the problem

$$
\left\{\begin{array}{c}
\left.\frac{\partial z^{\epsilon}}{\partial t}-\frac{1}{\epsilon} \nabla \cdot\left(\mathcal{A}^{\epsilon}(t, .) \nabla z^{\epsilon}\right)=\frac{1}{\epsilon}\left(\nabla \cdot \mathcal{C}^{\epsilon}(t, .)+\chi_{w} h^{\epsilon}\right), \text { in }\right] 0, T\left[\times \mathbb{T}^{2}\right. \\
z^{\epsilon}(x, 0)=z_{0}(x) \text { in } \Omega
\end{array}\right.
$$

where $f_{\epsilon} \in L^{\infty}\left((0, T), L^{2}(\Omega)\right)$ and $h^{\epsilon}$ is the control function and $z_{0} \in L^{2}(\Omega), \chi_{\omega}$ represent the charasteristic function of $\omega \subset \mathbb{T}^{2}$.

We consider also the following controlled problem,

$$
\frac{\partial U}{\partial \theta}-\nabla \cdot(\widetilde{\mathcal{A}}(t, . . .) \nabla U)=\nabla \cdot\left(\widetilde{\mathcal{C}}(t, ., .)+\mathbb{I}_{w} h(U)\right) .
$$

Before going further, we will recall the following notions of controllability.

Definition 1.1. [14] We say that (3) resp (4) is null controllable at time $T$, for each $z_{0} \in L^{2}\left(\mathbb{T}^{2}\right)$ initial data if there exists, a control $h^{\epsilon} \in L^{2}\left([0, T), L^{2}\left(\mathbb{T}^{2}\right)\right)$ (resp) $h \in$ $L^{\infty}\left([0, T), L_{\#}^{2}\left(\mathbb{R}, L^{2}\left(\mathbb{T}^{2}\right)\right)\right)$ such that the solutions $z^{\epsilon}$ to (3) resp $U$ to (4) satisfies $z^{\epsilon}(T, x)=0$ and $\operatorname{resp} U(t, 1, x)=0$.

Definition 1.2. [14] We say that (3) resp (4) is null locally controllable at time $T$, if there exists $\delta_{T}$ positif, for each $z_{0} \in L^{2}\left(\mathbb{T}^{2}\right)$, initial data verifiying $\left\|z_{0}\right\|_{L^{\infty}}<\delta_{T}$, there exists, a control $h^{\epsilon} \in L^{2}\left([0, T), L^{2}\left(\mathbb{T}^{2}\right)\right)$ (resp) $h \in$ $L^{\infty}\left([0, T), L_{\#}^{2}\left(\mathbb{R}, L^{2}\left(\mathbb{T}^{2}\right)\right)\right)$ such that the solutions $z^{\epsilon}$ to (3) resp $U$ to (4) satisfies $z^{\epsilon}(T, x)=0$ and $\operatorname{resp} U(t, 1, x)=0$.

\subsection{On the Control Problems}

In this paper, we will consider a null controllability result for the systems

$$
\left\{\begin{array}{c}
\frac{\partial U}{\partial \theta}-\nabla \cdot(\widetilde{\mathcal{A}}(t, ., .) \nabla U)=\nabla \cdot\left(\widetilde{\mathcal{C}}(t, ., .)+\chi_{w} h(U)\right),(\theta, t, x) \in(0,1) \times[0, T) \times \mathbb{T}^{2} \\
U(0,1, x)=U(0,0, X)=z_{0}(x) x \in \mathbb{T}^{2}
\end{array}\right.
$$

and

$$
\left\{\begin{array}{c}
\frac{\partial z^{\epsilon}}{\partial t}-\frac{1}{\epsilon} \nabla \cdot\left(\widetilde{\mathcal{A}}^{\epsilon}(t, ., .) \nabla z^{\epsilon}\right)=\frac{1}{\epsilon}\left(\nabla \cdot \widetilde{\mathcal{C}}^{\epsilon}(t, . . .)+\chi_{w} h^{\epsilon}(U)\right),(t, x) \in[0, T) \times \mathbb{T}^{2} \\
z^{\epsilon} / t=0=z_{0}(x), x \in \mathbb{T}^{2} \\
z^{\epsilon}(T, x)=z_{1}(x), x \in \mathbb{T}^{2}
\end{array}\right.
$$

where $T>0$ is given, $z_{0}, z_{1} \in L^{2}(\Omega)$ are the initial and final data. Moreover, $h$ and $h^{\epsilon}$ are locally distributed control acting on the control region $\omega \subset \Omega$.

The aim of this paper is to analyze the null controllability of equations (5) and (6). In perspective, we will look for the behavior of $h^{\epsilon}(t, x, U)$ in system (6) when $\epsilon$ goes to 0 .

\subsection{Presentation of the Results}

In this section, we analyze the null controllability, using a locally distributed control acting on the control region $\omega$.
We assume that $\omega$ is bounded open set included in the two dimensional torus $\mathbb{T}^{2}$. In the following, we consider that $\widetilde{\mathcal{A}} \in L^{\infty}\left([0, T], L_{\#}^{\infty}\left(\mathbb{R}, L^{2}\left(\mathbb{T}^{2}\right)\right)\right)$ and $\widetilde{\mathcal{C}} \in$ $\left(L^{\infty}\left([0, T], L_{\#}^{\infty}\left(\mathbb{R}, L^{2}\left(\mathbb{T}^{2}\right)\right)\right)\right)^{2}$ are respectively the twoscale limits of $\mathcal{A}^{\epsilon}(t, x)=\widetilde{\mathcal{A}}_{\epsilon}\left(t, \frac{t}{\epsilon}, x\right)$ and $\mathcal{C}^{\epsilon}(t, x)=$ $\widetilde{\mathcal{C}}_{\epsilon}\left(t, \frac{t}{\epsilon}, x\right)$. The coefficients $\widetilde{\mathcal{A}}_{\epsilon}$ and $\widetilde{\mathcal{C}}_{\epsilon}$ satisfy the following hypotheses

$$
\left\{\begin{array}{c}
\theta \longmapsto\left(\widetilde{\mathcal{A}}_{\epsilon}, \widetilde{\mathcal{C}}_{\epsilon}\right) \text { is periodic of period } 1 \\
x \longmapsto\left(\widetilde{\mathcal{A}}_{\epsilon}, \widetilde{\mathcal{C}}_{\epsilon}\right) \text { is defined on } \mathbb{T}^{2}, \\
\left|\widetilde{\mathcal{A}}_{\epsilon}\right| \leq \gamma,\left|\widetilde{\mathcal{C}}_{\epsilon}\right| \leq \gamma,\left|\frac{\partial \widetilde{\mathcal{A}}_{\epsilon}}{\partial t}\right| \leq \epsilon^{2} \gamma,\left|\frac{\partial \widetilde{\mathcal{C}}_{\epsilon}}{\partial t}\right| \leq \epsilon^{2} \gamma,\left|\frac{\partial \nabla \widetilde{\mathcal{A}}_{\epsilon}}{\partial t}\right| \leq \epsilon^{2} \gamma, \\
\left|\frac{\partial \widetilde{\mathcal{A}}_{\epsilon}}{\partial \theta}\right| \leq \gamma,\left|\frac{\partial \widetilde{\mathcal{C}}_{\epsilon}}{\partial \theta}\right| \leq \gamma,\left|\nabla \widetilde{\mathcal{A}}_{\epsilon}\right| \leq \epsilon \gamma,\left|\nabla \cdot \widetilde{\mathcal{C}}_{\epsilon}\right| \leq \epsilon \gamma,\left|\frac{\partial \nabla \cdot \widetilde{\mathcal{C}}_{\epsilon}}{\partial t}\right| \leq \epsilon^{2} \gamma
\end{array}\right.
$$




$$
\left\{\begin{array}{c}
\exists \widetilde{G}_{t h r}>0, \theta_{\alpha}<\theta_{\omega} \in[0,1] \text { not depending on } \epsilon \text { such that } \theta \in\left[\theta_{\alpha}, \theta_{\omega}\right] \Longrightarrow \widetilde{\mathcal{A}}_{\epsilon}(t, \theta, x) \geq \widetilde{G}_{t h r}, \\
\widetilde{\mathcal{A}}_{\epsilon}(t, \theta, x) \leq \widetilde{G}_{t h r} \Longrightarrow\left\{\begin{array}{l}
\frac{\partial \widetilde{\mathcal{A}}_{\epsilon}}{\partial t}(t, \theta, x)=0, \nabla \widetilde{\mathcal{A}}_{\epsilon}(t, \theta, x)=0 \\
\frac{\partial \widetilde{\mathcal{C}}_{\epsilon}}{\partial t}(t, \theta, x)=0, \nabla \cdot \widetilde{\mathcal{C}}_{\epsilon}(t, \theta, x)=0
\end{array}\right.
\end{array}\right.
$$

and

$$
\left\{\begin{array}{c}
\left|\widetilde{\mathcal{C}}_{\epsilon}\right| \leq \gamma\left|\widetilde{\mathcal{A}}_{\epsilon}\right|,\left|\widetilde{\mathcal{C}}_{\epsilon}\right|^{2} \leq \gamma\left|\widetilde{\mathcal{A}}_{\epsilon}\right|,\left|\nabla \widetilde{\mathcal{A}}_{\epsilon}\right| \leq \epsilon \gamma\left|\widetilde{\mathcal{A}}_{\epsilon}\right|,\left|\frac{\partial \widetilde{\mathcal{A}}_{\epsilon}}{\partial t}\right| \leq \epsilon^{2} \gamma\left|\widetilde{\mathcal{A}}_{\epsilon}\right|, \\
\left|\frac{\partial\left(\nabla \widetilde{\mathcal{A}}_{\epsilon}\right)}{\partial t}\right|^{2} \leq \epsilon^{2} \gamma\left|\widetilde{\mathcal{A}}_{\epsilon}\right|,\left|\nabla \cdot \widetilde{\mathcal{C}}_{\epsilon}\right| \leq \epsilon \gamma\left|\widetilde{\mathcal{A}}_{\epsilon}\right|,\left|\frac{\partial \widetilde{\mathcal{C}}_{\epsilon}}{\partial t}\right| \leq \epsilon^{2} \gamma\left|\widetilde{\mathcal{A}}_{\epsilon}\right|,\left|\frac{\partial \widetilde{\mathcal{C}}_{\epsilon}}{\partial t}\right|^{2} \leq \epsilon^{2} \gamma^{2}\left|\widetilde{\mathcal{A}}_{\epsilon}\right|,
\end{array}\right.
$$

where $\gamma$ and $\widetilde{\mathcal{G}}_{t h r}$ are constant positifs, not depending on $\epsilon$.

For the notion of two scales limits, we refer to $[1,9,16]$

We have the following theorem.

Theorem 1.1. Let $\epsilon>0$, for any $T>0$, under hypotheses (7)-(9), there exists a unique solution $z^{\epsilon} \in L^{2}\left([0, T), L^{2}\left(\mathbb{T}^{2}\right)\right)$ solution to (1). This solution satisfies

$$
\left\|z^{\epsilon}\right\|_{L^{2}\left([0, T), L^{2}(\Omega)\right)} \leq \gamma
$$

where $\gamma$ is a constant not depending on $\epsilon$.

Moreover, the solution $z^{\epsilon}$ to (1) two scales converges to $U \in L^{\infty}\left([0, T), L_{\#}^{2}\left(\mathbb{R}, L^{2}\left(\mathbb{T}^{2}\right)\right)\right)$ unique solution to (2).

Proof The proof of this theorem is done [9].

We have also the following theorem

Theorem 1.2. Let $\omega \subset \mathbb{T}^{2}$ and $\epsilon>0$, under assumptions (7)(9), the system (5) is null controllable at any time $T>0$. In other words, there exists a control $h^{\epsilon} \in L^{2}\left([0, T) \times \mathbb{T}^{2}\right)$ such that the solution $z^{\epsilon}$ to (6) satisfies $z^{\epsilon}(T, \cdot)=0$ in $L^{2}\left(\mathbb{T}^{2}\right)$.

We have our second null controllability result via the following theorem

Theorem 1.3. Assume that $\widetilde{\mathcal{A}}$ and $\widetilde{\mathcal{C}}$ are the two scales limits of $\mathcal{A}^{\epsilon}$ and $\mathcal{C}^{\epsilon}$ satisfying (7)-(9), there exists $h \in$ $L_{\#}^{\infty}\left(\mathbb{R}, L^{2}\left(\mathbb{R}, \mathbb{T}^{2}\right)\right)$ such that the solution $U$ to (5) satisfies $U(\theta, \cdot, \cdot)=0$ in $\left.(0, T) \times \mathbb{T}^{2}\right)$.

$$
\int_{\mathbb{T}^{2}}|w(t, 0, x)|^{2} d x \leq C_{0}\left(\mathbb{T}^{2}, \omega, \gamma, \widetilde{\mathcal{G}}_{t h}\right) \int_{0}^{T} \int_{\omega} w(t, \theta, x)^{2} d x d \theta .
$$

where $w$ is solution to the adjoint problem (5).

In the same way, assuming that hypotheses (7)-(9) holds, and considering $y^{\epsilon}$ the solution to the adjoint problem (12) we have

$$
\int_{\mathbb{T}^{2}}\left|w^{\epsilon}(0, x)\right|^{2} d x \leq C_{0}\left(\mathbb{T}^{2}, \omega, \gamma, \widetilde{\mathcal{G}}_{t h}\right) \int_{0}^{T} \int_{\omega} w^{\epsilon}(t, x)^{2} d x d t .
$$

\section{Inequality of Observability}

\subsection{Proof of Theorem 1.4}

As is classical in controllability theory, the result of theorem 1.4 can be given a dual form, introducing the so- called adjoint system of (5)

$$
\left\{\begin{array}{c}
\frac{\partial w}{\partial \theta}+\nabla \cdot(\widetilde{\mathcal{A}}(t, ., .) \nabla w)=0 \text { in }[0, T) \times \mathbb{R} \times \mathbb{T}^{2} \\
w(t, 0, x)=w(t, 1, x),(t, x) \in(0, T) \times \mathbb{T}^{2} \\
w(0,0, x)=z_{0}(x), x \in \mathbb{T}^{2}
\end{array}\right.
$$

In this equation, $t$ is only a parameter. The function $\theta \rightarrow$ $w(t, \theta, x)$ is 1 periodic.

The null controllability of (5) is equivalent to the following observability of the adjoint problem

Theorem 2.1. Let $\widetilde{\mathcal{A}}$ and $\widetilde{\mathcal{C}}$ be the two scale limits of $\mathcal{A}^{\epsilon}$ and $\mathcal{C}^{\epsilon}$. Let $T>0$, be given and $\omega \subset \mathbb{T}^{2}$, then there is a constant $C_{0}\left(\mathbb{T}^{2}, \omega, \gamma, \widetilde{\mathcal{G}}_{t h}\right)$ such that the solution $w \in$ $L^{\infty}\left([0, T), L_{\#}^{\infty}\left(\mathbb{R}, L^{2}\left(\mathbb{T}^{2}\right)\right)\right.$ to ( 15$)$ satisfies the following observability result

$$
\int_{\mathbb{T}^{2}}|w(t, 0, x)|^{2} d x \leq C_{0}\left(\mathbb{T}^{2}, \omega, \gamma, \widetilde{\mathcal{G}}_{t h}\right) \int_{0}^{T} \int_{\omega} w(t, \theta, x)^{2} d x d \theta .
$$


Proof Multiplying (15) by $w$ and integrating over the $\mathbb{T}^{2}$, we get

$$
\frac{1}{2} \frac{d}{d \theta} \int_{\mathbb{T}^{2}} w^{2} d x=\int_{\mathbb{T}^{2}} \widetilde{\mathcal{A}}(t, ., .)|\nabla w|^{2} \geq 0
$$

Because of the fact that, the second terme is positive, we get

$$
\frac{1}{2} \frac{d}{d \theta} \int_{\mathbb{T}^{2}} w^{2} d x \leq 0
$$

proving that the application $t \rightarrow \int_{\mathbb{T}^{2}} w^{2}(t, \theta, x) d x$ is nondecreasing.

Then we have,

$$
\frac{1}{2} \int_{\mathbb{T}^{2}} w^{2}(t, 0, x) d x-\frac{1}{2} \int_{\mathbb{T}^{2}} w^{2}(t, \theta, x) d x \leq 0 .
$$

Integrating from from $\frac{\theta_{1}}{4}$ to $\frac{3 \theta_{1}}{4}, \theta_{1} \in[0,1]$ with respect to $\theta$, we have

$$
\int_{\mathbb{T}^{2}} w(t, 0, x)^{2} d x \leq \frac{2}{\theta_{1}} \int_{\frac{\theta_{1}}{4}}^{\frac{3 \theta_{1}}{4}} \int_{\mathbb{T}^{2}} w(t, \theta, x)^{2} d x d \theta
$$

Let $R, \sigma$ and $\rho$ given as follows,

$$
\sigma(\theta, x)=\widetilde{\theta}(\theta)\left(e^{2 s\|\varphi\|_{\infty}}-e^{s \varphi(x)}\right) \text { and } \rho(t, x)=r s \tilde{\theta}(t) e^{s \varphi(x)}
$$

where

$$
\forall \theta \in\left(0, \theta_{1}\right), \tilde{\theta}(\theta)=\left(\frac{1}{\theta\left(\theta_{1}-\theta\right)}\right)^{k}
$$

then, the following equality holds

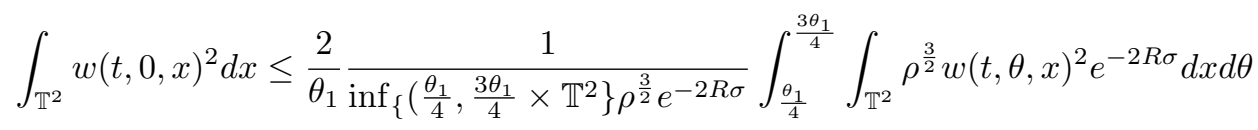

Following the idea developed by Cannarsa et al [2], there exists a constant $C$ depending on $\mathbb{T}^{2}$ such that for all $\theta_{1} \in[0,1]$

$$
\int_{\frac{\theta_{1}}{4}}^{\frac{3 \theta_{1}}{4}} \int_{\mathbb{T}^{2}} \rho^{\frac{3}{2}} w(t, \theta, x)^{2} d x d \theta \leq C \int_{\frac{\theta_{1}}{4}}^{\frac{3 \theta_{1}}{4}} \int_{\mathbb{T}^{2}} \rho^{2} w(t, \theta, x)^{2} e^{-2 R \sigma} d x d \theta
$$

Hence, we get

$$
\begin{gathered}
\int_{\mathbb{T}^{2}} w(t, 0, x)^{2} d x \leq \frac{2}{\theta_{1}} C \frac{1}{\left.\inf _{\{} \frac{\theta_{1}}{4}, \frac{3 \theta_{1}}{4} \times \mathbb{T}^{2}\right\} \rho^{\frac{3}{2}} e^{-2 R \sigma}} \int_{\frac{\theta_{1}}{4}}^{\frac{3 \theta_{1}}{4}} \int_{\mathbb{T}^{2}} \rho^{3} w(t, \theta, x)^{2} e^{-2 R \sigma} d x d \theta \\
\leq C \int_{0}^{\theta} \int_{\omega} v^{2}(t, \theta, x) d \theta d x
\end{gathered}
$$

We consider also, the adjoint state of (2)

$$
\left\{\begin{array}{c}
\frac{\partial y^{\epsilon}}{\partial t}+\nabla \cdot\left(\mathcal{A}^{\epsilon} \nabla y^{\epsilon}\right)=0,(t, x) \in Q_{T}=(0, T) \times \mathbb{T}^{2} \\
y^{\epsilon}(x, 0)=z_{0}(x), x \in \mathbb{T}^{2} \\
y^{\epsilon}(x, T)=z_{0}^{1}, x \in \mathbb{T}^{2}
\end{array}\right.
$$

We have also, the following lemma

Theorem 2.2. Let $T>0$ and $\epsilon>0$, under assumptions (7)-(9), there exists a constant $C_{0}\left(\mathbb{T}^{2}, \omega, \gamma, \widetilde{\mathcal{G}}_{t h}\right)$ such that the solution $y^{\epsilon}$ to (24) satisfies the following equality

$$
\int_{\mathbb{T}^{2}}\left|y^{\epsilon}(0, x)\right|^{2} d x \leq C_{0}\left(\mathbb{T}^{2}, \omega, \gamma, \widetilde{\mathcal{G}}_{t h}\right) \int_{0}^{T} \int_{\omega} y^{\epsilon}(t, x)^{2} d x d t .
$$


Proof We proceed in the same way, as in the proof of the above theorem. Multiplying equation (24) by $y^{\epsilon}$ and integrating over $\mathbb{T}^{2}$ we get

$$
\frac{1}{2} \frac{d}{d t} \int_{\mathbb{T}^{2}}\left(y^{\epsilon}\right)^{2}(t, x) d x=\frac{1}{\epsilon} \int_{\mathbb{T}^{2}} \mathcal{A}^{\epsilon}(t, x)\left|\nabla y^{\epsilon}\right|^{2} d x \geq 0
$$

As the second term is positive, we get from the last equality the following equality

$$
\frac{1}{2} \frac{d}{d t} \int_{\mathbb{T}^{2}}\left(y^{\epsilon}\right)^{2}(t, x) d x \geq 0
$$

Hence, the application $t \rightarrow \int_{\mathbb{T}^{2}}\left(y^{\epsilon}\right)^{2} d x$ is nondecreasing and we have

$$
\int_{\mathbb{T}^{2}}\left(y^{\epsilon}\right)^{2}(0, x) d x \leq \int_{\mathbb{T}^{2}}\left(y^{\epsilon}\right)^{2}(t, x) d x
$$

giving

$$
\begin{gathered}
\int_{\mathbb{T}^{2}}\left(y^{\epsilon}\right)^{2}(0, x) d x \leq \frac{2}{T} \int_{\frac{T}{4}}^{\frac{3 T}{4}} \int_{\mathbb{T}^{2}}\left(y^{\epsilon}\right)^{2}(t, x) d x d t \\
\leq \frac{2}{T} \frac{1}{\left.\inf _{\{}\left(\frac{T}{4}, \frac{3 T}{4}\right) \times \mathbb{T}^{2}\right\} \rho^{\frac{3}{2}} e^{-2 R \sigma}} \int_{0}^{T} \int_{\mathbb{T}^{2}} \rho^{3}\left(y^{\epsilon}\right)^{2}(t, x) e^{-2 R \sigma} d x d t,
\end{gathered}
$$

where $R, \sigma$ and $\rho$ are given in the proof of the above thoerem. Using a result Cannarsa et al.[2] recalled in the proof of the above theorem, we

$$
\int_{\mathbb{T}^{2}}\left(y^{\epsilon}\right)^{2}(0, x) d x \leq \frac{2}{T} \frac{1}{\left.\inf _{\{}\left(\frac{T}{4}, \frac{3 T}{4}\right) \times \mathbb{T}^{2}\right\} \rho^{\frac{3}{2}} e^{-2 R \sigma}} C \int_{0}^{T} \int_{\mathbb{T}^{2}} \rho^{3}\left(y^{\epsilon}\right)^{2}(t, x) e^{-2 R \sigma} d x d t, \leq C \int_{0}^{T} \int_{\omega}\left(y^{\epsilon}\right)^{2} d x d t
$$

where $C$ depend on the domain $\mathbb{T}^{2}, \omega$ and $T$.

\subsection{Equivalence Between Null Controllability and Observability}

This section is devoted to the proof of theorem 1.2 and theorem 1.3. Thus, in other words, (5) and (6) are null controllable. Suppose (5) is null controllable and the control $h$ is bounded. Let $U$ be the solution of (5) and let $h \in L^{2}\left((0, T), L^{2}(\Omega) \in L^{2}(\Omega)\right.$ be a control steering the solution $U$ of (5) with $U(0,0, \cdot)=z_{0}(x)$ such that

$$
\int_{\mathbb{T}^{2}} \int_{0}^{1}|h(t, \theta, x)|^{2} d x d \theta \leq C\left(\mathbb{T}^{2}, \omega, T\right) \int_{\mathbb{T}^{2}}|w(0,0, x)|^{2} d x
$$

Then, multiplying (5) by $w$ and (24) by $U$, and integrating by parts over $\mathbb{T}^{2}$ leads to

$$
\begin{gathered}
\int_{\mathbb{T}^{2}} \frac{\partial U}{\partial \theta} w d x+\int_{\mathbb{T}^{2}} \widetilde{\mathcal{A}} \nabla U \cdot \nabla w d x=\int_{\omega} h w d x \\
\int_{\mathbb{T}^{2}} \frac{\partial w}{\partial \theta} U d x-\int_{\mathbb{T}^{2}} \widetilde{\mathcal{A}} \nabla w \cdot \nabla U d x=0,
\end{gathered}
$$

then, we get, by summing the two expressions

$$
\frac{d}{d \theta} \int_{\mathbb{T}^{2}} U w d x=\int_{\omega} h w d x .
$$

Integrating from 0 to $\theta_{1} \in[0, T]$, and taking into account that $U\left(t, \theta_{1}, 0\right)=0$ we get

$$
\begin{gathered}
\int_{\mathbb{T}^{2}}|w(0, x)|^{2}=\left|\int_{0}^{1} \int_{\mathbb{T}^{2}} h v d x d \theta\right| \\
\leq C^{1 / 2}\left(\int_{\mathbb{T}^{2}}|w(0,0, x)|^{2} d x\right)^{1 / 2}\left(\int_{0}^{1} \int_{\mathbb{T}^{2}}|w(t, \theta, x)|^{2} d x d \theta\right)^{1 / 2}
\end{gathered}
$$


giving the result.

Conversely, assume that we have an observability equality for the solution $w$ to (15) and let $z_{0} \in L^{2}\left(\mathbb{T}^{2}\right)$.

For any $\epsilon>0$, we consider the functional

$$
J_{\epsilon}(h)=\frac{1}{2} \int_{0}^{1} \int_{\mathbb{T}^{2}}|h|^{2} d x d \theta+\frac{1}{2 \epsilon} \int_{\mathbb{T}^{2}}\left|u^{h}(1, x)\right|^{2} d x,
$$

where, for every $h \in L^{2}\left(\mathbb{R} \times \mathbb{T}^{2}\right), u^{h}$ denotes the corresponding solution of (5). By a straightforward convexity argument, $J_{\epsilon}$ attains its minimum at a unique point, say $h_{\epsilon} \in L^{2}\left(\mathbb{R} \times \mathbb{T}^{2}\right)$. Then, writing $u^{\epsilon}$ for $u^{h^{\epsilon}}$, Fermat's rule yields, for all $g \in L^{2}(\Omega)$,

$$
\iint_{\mathbb{T}^{2}} h^{\epsilon} g d x d t+\frac{1}{\epsilon} \int_{\mathbb{T}^{2}} u^{\epsilon}(T, x) U^{g}(, x) d x=0
$$

where $U^{g}$ is the solution of the problem

$$
\left\{\begin{array}{l}
\frac{\partial U}{\partial \theta}-\nabla \cdot(\mathcal{A} \nabla U)=g \chi_{\omega}(t, \theta, x) \text { in }(0, T) \times \mathbb{R} \times \mathbb{T}^{2} \\
U(0,0, x)=0 x \in \mathbb{T}^{2} .
\end{array}\right.
$$

Now, let $v^{\epsilon}$ be the solution of (15) with

$$
v_{1}(x)=\frac{1}{\epsilon} U^{\epsilon}(1, x), x \in \mathbb{T}^{2}
$$

and multiplying (36) by $v^{\epsilon}$ and (15) by $U^{g}$ we get

$$
\frac{d}{d \theta} \int_{\mathbb{T}^{2}} U^{g} v^{\epsilon} d x=\int_{\omega} v^{\epsilon} g d x d t
$$

Hence

$$
\frac{1}{\epsilon} \int_{\mathbb{T}^{2}} U^{\epsilon}(1, x) U^{g}(1, x) d x=\int_{0}^{1} \int_{\omega} v^{\epsilon} g d x d t
$$

which, combined with (35), implies that $h^{\epsilon}=-\chi_{\omega} v^{\epsilon}$. Therefore, by a same argument, we get from (36) and (15),

$$
\frac{d}{d t} \int_{\mathbb{T}^{2}} U^{\epsilon} v^{\epsilon} d x=-\int_{\omega}\left|v^{\epsilon}\right|^{2} d x
$$

Then, integrating over $[0,1]$ and recalling that $w^{\epsilon}(1, x)=\frac{1}{\epsilon} U^{\epsilon}(1, x)$, we get

$$
\frac{1}{\epsilon} \int_{\mathbb{T}^{2}}\left|U^{\epsilon}(1, x)\right|^{2} d x+\int_{0}^{1} \int_{\omega}\left|v^{\epsilon}\right|^{2} d x d t=\int_{\mathbb{T}^{2}} u_{0}(x) v^{\epsilon}(0, x) d x .
$$

Thus, using observability inequality (25) to bound the $L^{2}$-norm of $v^{\epsilon}(0,$.$) , one obtains$

$$
\frac{1}{\epsilon} \int_{\mathbb{T}^{2}}\left|u^{\epsilon}(1, x)\right|^{2} d x+\frac{1}{2} \int_{0}^{1} \int_{\omega}\left|v^{\epsilon}\right|^{2} d x d t \leq C \int_{\mathbb{T}^{2}}\left|u_{0}(x)\right|^{2} d x,
$$

where $C=C(\mathbb{T}, \omega, 1)$. Since

$$
h^{\epsilon}=-\chi_{\omega} v^{\epsilon}
$$

the last inequality reads as

$$
\frac{1}{\epsilon} \int_{\mathbb{T}^{2}}\left|U^{\epsilon}(1, x)\right|^{2} d x+\frac{1}{2} \iint_{\omega}\left|v^{\epsilon}\right|^{2} d x d t \leq \int_{\mathbb{T}^{2}}\left|u_{0}(x)\right|^{2} d x,
$$

So, the weak limit, say $h^{0}$, of $h^{\epsilon_{i}}$ along a suitable sequence $\epsilon \rightarrow 0$ satisfies (5).

\section{Carlman Estimate}

In this section, we give Carleman estimate of the solution $W$ solution to (5). Carleman estimates are weighted Sobolev inequalites satisfied by the solution. For all $s>0$, let us define the weight function $\varphi(\theta, x)$ such that

$$
W(t, \theta, x)=e^{s \varphi(\theta, x)} U(t, \theta, x),
$$


where $U$ is solution (5). Then we have

$$
U(t, \theta, x)=e^{-s \varphi(\theta, x)} W(t, \theta, x)
$$

Replacing $U$ in (5), we get

$$
\frac{\partial\left(e^{-s \varphi} W\right)}{\partial \theta}-\nabla \cdot\left(\widetilde{\mathcal{A}} \nabla\left(e^{-s \varphi} W\right)\right)=\nabla \cdot(\widetilde{\mathcal{C}})+\chi_{w} h(U) .
$$

In the following, we recalculate, based on data, all the terms of the equation (39) in order to reconstruct it. Because of this, we get

$$
\frac{\partial\left(e^{-s \varphi} W\right)}{\partial \theta}=\left[-s \frac{\partial \varphi}{\partial \theta} W+\frac{\partial W}{\partial \theta}\right] e^{-s \varphi}
$$

and

$$
\tilde{\mathcal{A}} \nabla\left(e^{-s \varphi} W(\theta, x)\right)=\tilde{\mathcal{A}}\left[-s \nabla \varphi e^{-s \varphi} W+e^{-s \varphi} \nabla W\right]
$$

giving

$$
\begin{gathered}
\nabla \cdot\left(\widetilde{\mathcal{A}} \nabla\left(e^{-s \varphi} W(\theta, x)\right)\right)=-s \nabla \widetilde{\mathcal{A}} \nabla \varphi e^{-s \varphi} W+\nabla \widetilde{\mathcal{A}} e^{-s \varphi} \nabla W-s \widetilde{\mathcal{A}} \Delta \varphi e^{-s \varphi} W \\
+s^{2} \widetilde{\mathcal{A}}|\nabla \varphi|^{2} e^{-s \varphi} w-2 s \tilde{A} \nabla \varphi \nabla W e^{-s \varphi}+e^{-s \varphi} \tilde{A} \Delta W .
\end{gathered}
$$

Then, equation (5) becomes

$$
\begin{gathered}
\frac{\partial U}{\partial \theta}-\nabla \cdot(\widetilde{\mathcal{A}} \nabla U)=\frac{\partial\left(e^{-s \varphi} W\right)}{\partial \theta}-\nabla \cdot\left(\widetilde{\mathcal{A}} \nabla\left(e^{-s \varphi} W\right)\right) \\
-s e^{-s \varphi}\left[-s \frac{\partial \varphi}{\partial \theta} W+\frac{\partial W}{\partial \theta}+\nabla \widetilde{\mathcal{A}} \nabla W-s^{2} \widetilde{\mathcal{A}}|\nabla \varphi|^{2} W+2 s \widetilde{\mathcal{A}} \nabla \varphi \nabla W-\nabla \cdot(\widetilde{\mathcal{A}} \nabla W) .\right]
\end{gathered}
$$

Then, the solution $W$ solves the following system

$$
\left\{\begin{array}{c}
\frac{\partial\left(e^{-s \varphi} W\right)}{\partial \theta}-\nabla \cdot\left(\widetilde{\mathcal{A}} \nabla\left(e^{-s \varphi} W\right)\right) \\
-s e^{-s \varphi}\left[-s \frac{\partial \varphi}{\partial \theta} W+\frac{\partial W}{\partial \theta}+\nabla \widetilde{\mathcal{A}} \nabla W-s^{2} \widetilde{\mathcal{A}}|\nabla \varphi|^{2} W+2 s \widetilde{\mathcal{A}} \nabla \varphi \nabla W-\nabla \cdot(\widetilde{\mathcal{A}} \nabla W)\right]=\nabla \cdot\left(\widetilde{\mathcal{C}}+\chi_{w} h(U)\right) . \\
W(t, \theta, x)=W(t, 1, x), x \in \mathbb{T}^{2}
\end{array}\right.
$$

In the following, let's define the following operator $P$ by

$$
P W=\frac{\partial W}{\partial \theta}-\nabla \cdot(\widetilde{\mathcal{A}} \nabla W)+2 s \widetilde{\mathcal{A}} \nabla \varphi \nabla W+\left(-s \frac{\partial \varphi}{\partial \theta}-s^{2}|\nabla \varphi|^{2} \widetilde{\mathcal{A}}+s \nabla \cdot(\widetilde{\mathcal{A}} \nabla \varphi)\right) W
$$

$W$ defined by (37) is solution to

$$
P W=e^{s \varphi} \nabla \cdot(\widetilde{\mathcal{C}}+h) \text { in } \mathbb{R} \times \mathbb{T}^{2} .
$$

We are now interested in the adjoint operator $P^{*}$ of $P$ defined as follows $(P W, V)=\left(W, P^{*} V\right)$.

We have

$$
\begin{gathered}
\left(\frac{\partial W}{\partial t}, V\right)=-\left(W, \frac{\partial V}{\partial t}\right) \\
(-\Delta W, V)=(W,-\Delta V)
\end{gathered}
$$

Multiplying (44) by $V$ and integrating, we get

$$
\begin{aligned}
& \left(\frac{\partial W}{\partial \theta} V-\widetilde{\mathcal{A}} \Delta W V-\nabla \widetilde{\mathcal{A}} \nabla W V+2 s \widetilde{\mathcal{A}} \nabla \varphi \nabla W V\right)+\left(-s \frac{\partial \varphi}{\partial \theta}-s^{2}|\nabla \varphi|^{2} \widetilde{\mathcal{A}}+s \widetilde{\mathcal{A}} \Delta \varphi+s \nabla \widetilde{\mathcal{A}} \nabla \varphi\right) W V \\
= & -W \frac{\partial V}{\partial \theta}-\widetilde{\mathcal{A}} W \Delta V+\nabla \widetilde{\mathcal{A}} \nabla V W-2 s \widetilde{\mathcal{A}} \nabla \varphi W \nabla V+\left(-s \frac{\partial \varphi}{\partial \theta}-s^{2}|\nabla \varphi|^{2} \widetilde{\mathcal{A}}+s \widetilde{\mathcal{A}} \Delta \varphi+s \nabla \widetilde{\mathcal{A}} \nabla \varphi\right) W V \\
= & W\left[-\frac{\partial V}{\partial \theta}-\widetilde{\mathcal{A}} \Delta V-2 s \widetilde{\mathcal{A}} \nabla \varphi \nabla V+\nabla \widetilde{\mathcal{A}} \nabla V+\left(-s \frac{\partial \varphi}{\partial \theta}-s^{2} \widetilde{\mathcal{A}}|\nabla \varphi|^{2}+s \widetilde{\mathcal{A}} \Delta \varphi+s \nabla \widetilde{\mathcal{A}} \nabla \varphi\right) V\right]
\end{aligned}
$$


Thus, the adjoint operator $P^{*} V$ is identified as follows:

$$
P^{*} V=-\frac{\partial V}{\partial \theta}-\nabla \cdot(\widetilde{\mathcal{A}} \nabla V)-2 s \widetilde{\mathcal{A}} \nabla \varphi \nabla V+\left(-s \frac{\partial \varphi}{\partial \theta}-s^{2} \widetilde{\mathcal{A}}|\nabla \varphi|^{2}-s \nabla \cdot(\widetilde{\mathcal{A}} \nabla \varphi)\right) V
$$

and we define the two operators $P^{+} W$ and $P^{-} W$, as the following

$$
\begin{aligned}
& P^{+} W=\frac{1}{2}\left[P W+P^{*} W\right] \\
& P^{-} W=\frac{1}{2}\left[P W-P^{*} W\right]
\end{aligned}
$$

giving directly

$$
P W=P^{+} W+P^{-} W=e^{s \varphi} \nabla \cdot(\widetilde{\mathcal{C}}+h) \text { in } \mathbb{R} \times \mathbb{T}^{2}
$$

From (44) and (48), we have

$$
\begin{gathered}
P^{+} W=\frac{1}{2}\left[\frac{\partial W}{\partial \theta}-\nabla \cdot(\widetilde{\mathcal{A}} \nabla W)+2 s \widetilde{\mathcal{A}} \nabla \varphi \nabla W+\left(-s \frac{\partial \varphi}{\partial \theta}-s^{2}|\nabla \varphi|^{2} \widetilde{\mathcal{A}}+s \nabla \cdot(\widetilde{\mathcal{A}} \nabla \varphi)\right) W\right. \\
\left.-\frac{\partial W}{\partial \theta}+\nabla \cdot(\widetilde{\mathcal{A}} \nabla W)-2 s \widetilde{\mathcal{A}} \nabla \varphi \nabla W+\left(-s \frac{\partial \varphi}{\partial \theta}-s^{2} \widetilde{\mathcal{A}}|\nabla \varphi|^{2}+s \nabla \cdot(\widetilde{\mathcal{A}} \nabla \varphi)\right) W\right] \\
P^{+} W=\left(-s \frac{\partial \varphi}{\partial \theta}-s^{2}|\nabla \varphi|^{2} \widetilde{\mathcal{A}}+s \nabla \cdot(\widetilde{\mathcal{A}} \nabla \varphi)\right) W
\end{gathered}
$$

and

$$
\begin{aligned}
P^{-} W= & \frac{1}{2}\left[\frac{\partial W}{\partial \theta}-\nabla \cdot(\widetilde{\mathcal{A}} \nabla W)+2 s \widetilde{\mathcal{A}} \nabla \varphi \nabla W+\left(-s \frac{\partial \varphi}{\partial \theta}-s^{2}|\nabla \varphi|^{2} \widetilde{\mathcal{A}}+s \nabla \cdot(\widetilde{\mathcal{A}} \nabla \varphi)\right) W\right. \\
& \left.+\frac{\partial W}{\partial \theta}+\nabla \cdot(\widetilde{\mathcal{A}} \nabla W)+2 s \widetilde{\mathcal{A}} \nabla \varphi \nabla W-\left(-s \frac{\partial \varphi}{\partial \theta}-s^{2} \widetilde{\mathcal{A}}|\nabla \varphi|^{2}+s \nabla \cdot(\widetilde{\mathcal{A}} \nabla \varphi)\right) W\right]
\end{aligned}
$$

from which we have

$$
\left.P^{-} W=\frac{\partial W}{\partial \theta}+2 s \widetilde{\mathcal{A}} \nabla \varphi \nabla W+s \nabla \cdot(\widetilde{\mathcal{A}} \nabla \varphi)\right) W
$$

We have the following lemma.

Lemma 3.1 Let $D=[0,1] \times \mathbb{T}^{2}$. The following identity holds

$$
\begin{aligned}
\left(P^{+} W, P^{-} W\right)_{L^{2}(D)} & =-s \int_{0}^{1} \int_{\mathbb{T}^{2}} \frac{\partial \varphi}{\partial \theta} W \frac{\partial W}{\partial \theta} d x d \theta-2 s^{2} \int_{0}^{1} \int_{\mathbb{T}^{2}} \frac{\partial \varphi}{\partial \theta} \nabla \varphi \widetilde{A} W \nabla W d x d \theta \\
& -s^{2} \int_{0}^{1} \int_{\mathbb{T}^{2}} \frac{\partial \varphi}{\partial \theta} \Delta \varphi \widetilde{A} W^{2} d x d \theta-s^{2} \int_{0}^{1} \int_{\mathbb{T}^{2}}|\nabla \varphi|^{2} \widetilde{A} W \frac{\partial W}{\partial \theta} d x d \theta \\
& -2 s^{3} \int_{0}^{1} \int_{\mathbb{T}^{2}}|\nabla \varphi|^{3}|\widetilde{\mathcal{A}}|^{2} W \nabla W d x d \theta-s^{3} \int_{0}^{1} \int_{\mathbb{T}^{2}}|\nabla \varphi|^{2} \Delta \varphi|\widetilde{\mathcal{A}}|^{2} W^{2} d x d \theta \\
& +s \int_{0}^{1} \int_{\mathbb{T}^{2}} \Delta \varphi \widetilde{\mathcal{A}} W \frac{\partial W}{\partial \theta} d x d \theta+2 s^{2} \int_{0}^{1} \int_{\mathbb{T}^{2}} \Delta \varphi \nabla \varphi|\widetilde{\mathcal{A}}|^{2} W \nabla W d x d \theta \\
& +\int_{0}^{1} \int_{\mathbb{T}^{2}}|\Delta \varphi|^{2}|\widetilde{\mathcal{A}}|^{2} W^{2} d x d \theta
\end{aligned}
$$

Proof We have the following equality

$$
\left|\left(P^{+} W+P^{-} W\right)\right|^{2}=\left|P^{+} W\right|^{2}+2<P^{+} W, P^{-} W>+\left\|P^{-} W\right\|^{2}
$$

Then we have,

$$
\|P W\|^{2}=\left\|P^{+} W\right\|^{2}+2<P^{+} W, P^{-} W>+\left\|P^{-} W\right\|^{2},
$$


and

$$
\begin{gathered}
2\left\langle P^{+} W, P^{-} W\right\rangle \leq\|P W\|^{2} \\
2<P^{+} W, P^{-} W>\leq\left\|e^{s \varphi}(\nabla \cdot \widehat{c}+h)\right\|^{2} .
\end{gathered}
$$

Replacing each operator by it's expression we have

$$
\left.\left(P^{+} W, P^{-} W\right)=\left(\left(-s \frac{\partial \varphi}{\partial \theta}-s^{2}|\nabla \varphi|^{2} \widetilde{\mathcal{A}}+s \nabla \cdot(\widetilde{\mathcal{A}} \nabla \varphi)\right) W, \frac{\partial W}{\partial \theta}+2 s \widetilde{\mathcal{A}} \nabla \varphi \nabla W+s \nabla \cdot(\widetilde{\mathcal{A}} \nabla \varphi)\right) W\right),
$$

and because of the linear of the scalar product we get directly.

$$
\left(P^{+} W, P^{-} W\right)=\underbrace{\left(\left(-s \frac{\partial \varphi}{\partial \theta}-s^{2}|\nabla \varphi|^{2} \widetilde{\mathcal{A}}+s \widetilde{\mathcal{A}} \Delta \varphi\right) W, \frac{\partial W}{\partial \theta}\right)}_{=I_{1}}+\underbrace{\left(P^{+} W, 2 s \widetilde{\mathcal{A}} \nabla \varphi \nabla W\right)}_{=I_{2}}+\underbrace{\left.\left(P^{+} W, s \nabla \cdot(\widetilde{\mathcal{A}} \nabla \varphi) W\right)\right)}_{=I_{3}} .
$$

Developing term by term we have,

$$
\begin{aligned}
& I_{1}=\left(\left(-s \frac{\partial \varphi}{\partial \theta}-s^{2}|\nabla \varphi|^{2} \widetilde{\mathcal{A}}+s \widetilde{\mathcal{A}} \Delta \varphi\right) W, \frac{\partial W}{\partial \theta}\right)=\left(-s \frac{\partial \varphi}{\partial \theta}-s^{2}|\nabla \varphi|^{2} \widetilde{\mathcal{A}}, \frac{\partial W}{\partial \theta}\right)_{L^{2}(D)}+\left((-s \widetilde{\mathcal{A}} \Delta \varphi) W, \frac{\partial W}{\partial \theta}\right)_{L^{2}(D)}, \\
& I_{2}=\left(P^{+} W, 2 s \widetilde{\mathcal{A}} \nabla \varphi \nabla W\right)_{L^{2}(D)}=\left(-s \frac{\partial \varphi}{\partial \theta}-s^{2}|\nabla \varphi|^{2} \widetilde{\mathcal{A}}, 2 s \widetilde{\mathcal{A}} \nabla \varphi \nabla W\right)_{L^{2}(D)}+(\nabla \cdot(\widetilde{\mathcal{A}} \nabla W), 2 s \widetilde{\mathcal{A}} \nabla \varphi \nabla W)_{L^{2}(D)},
\end{aligned}
$$

and

$$
\begin{gathered}
I_{3}=\left(P^{+} W, s \nabla \cdot(\widetilde{\mathcal{A}} \nabla \varphi) W\right)_{L^{2}(D)}=\left(\left(-s \frac{\partial \varphi}{\partial \theta}-s^{2}|\nabla \varphi|^{2} \widetilde{\mathcal{A}}\right) W, s \nabla \cdot(\widetilde{\mathcal{A}} \nabla \varphi) W\right)_{L^{2}(D)} \\
+(\nabla \cdot(\widetilde{\mathcal{A}} \nabla W), s \nabla \cdot(\widetilde{\mathcal{A}} \nabla \varphi) W)_{L^{2}(D)} .
\end{gathered}
$$

Combining the formulas giving in (55), (56) and (57), we get for $\left(P^{+} W, P^{-} W\right)_{L^{2}(D)}$ the following expression

$$
\begin{gathered}
\left(P^{+} W, P^{-} W\right)_{L^{2}(D)}=\left(\left(-s \frac{\partial \varphi}{\partial \theta}-s^{2}|\nabla \varphi|^{2} \widetilde{\mathcal{A}}\right) W, \frac{\partial W}{\partial \theta}\right)_{L^{2}(D)}+\left((-s \widetilde{\mathcal{A}} \Delta \varphi) W, \frac{\partial W}{\partial \theta}\right)_{L^{2}(D)} \\
+\left(\left(-s \frac{\partial \varphi}{\partial \theta}-s^{2}|\nabla \varphi|^{2} \widetilde{\mathcal{A}}\right) W, 2 s \widetilde{\mathcal{A}} \nabla \varphi \nabla W\right)_{L^{2}(D)}+(\nabla \cdot(\widetilde{\mathcal{A}} \nabla W), 2 s \widetilde{\mathcal{A}} \nabla \varphi \nabla W)_{L^{2}(D)} \\
+\left(\left(-s \frac{\partial \varphi}{\partial \theta}-s^{2}|\nabla \varphi|^{2} \widetilde{\mathcal{A}}\right) W, s \nabla \cdot(\widetilde{\mathcal{A}} \nabla \varphi) W\right)_{L^{2}(D)}+(\nabla \cdot(\widetilde{\mathcal{A}} \nabla W), s \nabla \cdot(\widetilde{\mathcal{A}} \nabla \varphi) W)_{L^{2}(D)}
\end{gathered}
$$

giving

$$
\begin{aligned}
\left(P^{+} W, P^{-} W\right) & =-s \int_{0}^{1} \int_{\mathbb{T}^{2}}\left(\frac{\partial \varphi}{\partial \theta} W\right) \frac{\partial W}{\partial \theta} d x d \theta-2 s^{2} \int_{0}^{1} \int_{\mathbb{T}^{2}}\left(\frac{\partial \varphi}{\partial \theta} W\right)(\widetilde{\mathcal{A}} \nabla \varphi \nabla W) d x d \theta \\
- & s^{2} \int_{0}^{1} \int_{\mathbb{T}^{2}}\left(\frac{\partial \varphi}{\partial \theta} W\right)(\widetilde{\mathcal{A}} \Delta \varphi W) d x d \theta-s^{2} \int_{0}^{1} \int_{\mathbb{T}^{2}}\left(|\nabla \varphi|^{2} \widetilde{\mathcal{A}} W \frac{\partial W}{\partial \theta}\right) d x d \theta \\
- & -s^{3} \int_{0}^{1} \int_{\mathbb{T}^{2}}\left(|\nabla \varphi|^{2} \widetilde{\mathcal{A}} W\right)(\widetilde{\mathcal{A}} \nabla \varphi \nabla W) d x d \theta-s^{3} \int_{0}^{1} \int_{\mathbb{T}^{2}}\left(|\nabla \varphi|^{2} \widetilde{\mathcal{A}} W\right)(\widetilde{\mathcal{A}} \Delta \varphi W) d x d \theta \\
& +s \int_{0}^{1} \int_{\mathbb{T}^{2}}(\widetilde{\mathcal{A}} \Delta \varphi W) \frac{\partial W}{\partial \theta} d x d \theta+2 s^{2} \int_{0}^{1} \int_{\mathbb{T}^{2}}(\widetilde{\mathcal{A}} \Delta \varphi W)(\widetilde{\mathcal{A}} \nabla \varphi \nabla W) d x d \theta
\end{aligned}
$$

Proposition 3.1 The following identity holds

$$
\left(P^{+} W, P^{-} W\right)_{L^{2}(D)}=\int_{\mathbb{T}^{2}}\left[\left(-s \frac{\partial \varphi}{\partial \theta}-s^{2} \widetilde{\mathcal{A}} \nabla|\varphi|^{2}\right) W^{2}\right]_{0}^{1} d x+s \int_{D} \frac{\partial^{2} \varphi}{\partial \theta^{2}} W^{2} d x d \theta+s^{2} \int_{D} \frac{\partial \widetilde{\mathcal{A}}}{\partial \theta}|\varphi|^{2} W^{2} d x d \theta
$$




$$
\begin{gathered}
+s^{2} \int_{D} \widetilde{\mathcal{A}} \frac{\partial}{\partial \theta}|\varphi|^{2} W^{2} d x d \theta+s \sum_{j} \int_{D} \widetilde{\mathcal{A}}\left[\frac{\partial}{\partial x_{j}}\left(\frac{\partial W}{\partial x_{j}} \frac{\partial W}{\partial \theta}\right)\right] d x d \theta+2 s \int_{D}\left[\nabla\left(s \frac{\partial \varphi}{\partial \theta}+s^{2}|\nabla \varphi|^{2} \widetilde{\mathcal{A}}\right)\right. \\
\left.+\left(s \frac{\partial \varphi}{\partial \theta}+s^{2}|\nabla \varphi|^{2} \nabla \widetilde{\mathcal{A}}\right)\right] \nabla \varphi W^{2} d x d \theta+2 s^{2} \int_{D} \frac{\partial \varphi}{\partial \theta} \widetilde{\mathcal{A}} \Delta \varphi W^{2} d x d \theta+2 s^{3} \int_{D} \widetilde{\mathcal{A}}^{2}|\nabla \varphi|^{2} \Delta \varphi W^{2} d x d \theta \\
+4 s \int_{D}\left(\widetilde{\mathcal{A}} \nabla \widetilde{\mathcal{A}}|\nabla W|^{2}+\widetilde{\mathcal{A}}^{2} \nabla \varphi \nabla W \Delta W\right) d x d \theta+s \int_{D}\left(-s \frac{\partial \varphi}{\partial \theta}-s^{2}|\nabla \varphi|^{2} \widetilde{\mathcal{A}}\right) \nabla \cdot(\widetilde{\mathcal{A}} \nabla \varphi) W^{2} d x d \theta \\
+s^{2} \int_{D}|\nabla \cdot(\widetilde{\mathcal{A}} \nabla \varphi)|^{2} W^{2} d x d \theta
\end{gathered}
$$

Proof It is enough to develop the integrals $I_{1}, I_{2}$ and $I_{3}$ and by simplifying some expressions to obtain the result. We also consider the fact that the function $\varphi(x, \theta)$ belongs to $\mathcal{C}^{\infty}\left([0,1] \times \mathbb{T}^{2}\right)$ and is 1-periodic with respect to the variable $\theta$. Following this idea, we have

$$
\begin{aligned}
I_{1} & =\left(P^{+}, \frac{\partial W}{\partial \theta}\right)=\left(\left(-s \frac{\partial \varphi}{\partial \theta}-s^{2}|\nabla \varphi|^{2} \widetilde{\mathcal{A}}+s \nabla \cdot(\tilde{\mathcal{A}} \nabla \varphi)\right) W, \frac{\partial W}{\partial \theta}\right) \\
& =\int_{D}\left(-s \frac{\partial \varphi}{\partial \theta}-s^{2}|\nabla \varphi|^{2} \widetilde{\mathcal{A}}\right) W \frac{\partial W}{\partial \theta} d x d \theta+\int_{D} s \nabla \cdot(\widetilde{\mathcal{A}} \nabla \varphi) W \frac{\partial W}{\partial \theta}
\end{aligned}
$$

Integrating by parts the integral $I_{1}$ with respect to the variable $\theta$, and using periodicity, we get

$$
\begin{aligned}
I_{1}=\int_{\mathbb{T}^{2}}\left[\left(-s \frac{\partial \varphi}{\partial \theta}-s^{2} \widetilde{\mathcal{A}} \nabla|\varphi|^{2}\right) W^{2}\right]_{0}^{1} d x+s \int_{D} \frac{\partial^{2} \varphi}{\partial \theta^{2}} W^{2} d x d \theta+s^{2} \int_{D} \frac{\partial \widetilde{\mathcal{A}}}{\partial \theta}|\varphi|^{2} W^{2} d x d \theta \\
+s^{2} \int_{D} \widetilde{\mathcal{A}} \frac{\partial}{\partial \theta}|\varphi|^{2} W^{2} d x d \theta+s \int_{D} \sum_{j} \frac{\partial}{\partial x_{j}}\left(\widetilde{\mathcal{A}} \frac{\partial \varphi}{\partial x_{j}}\right) W \frac{\partial W}{\partial \theta} d x d \theta
\end{aligned}
$$

The last term of (58) can be written as follow

$$
J_{2}=s \sum_{j} \int_{D}\left[\frac{\partial \widetilde{\mathcal{A}}}{\partial x_{j}} \frac{\partial \varphi}{\partial x_{j}} W \frac{\partial W}{\partial \theta}+\widetilde{\mathcal{A}} \frac{\partial}{\partial x_{j}}\left(\frac{\partial \varphi}{\partial x_{j}}\right) W \frac{\partial W}{\partial \theta}\right] d x d \theta
$$

Integrating $J_{2}$ by parts in the first term, we have

$$
J_{2}=\sum_{j} \int_{D} \widetilde{\mathcal{A}}\left[\frac{\partial}{\partial x_{j}}\left(\frac{\partial \varphi}{\partial x_{j}} W \frac{\partial W}{\partial \theta}\right)+\frac{\partial}{\partial x_{j}}\left(\frac{\partial \varphi}{\partial x_{j}}\right) W \frac{\partial W}{\partial \theta}\right] d x d \theta
$$

and then,

$$
J_{2}=s \int_{D} \widetilde{\mathcal{A}} \Delta \varphi \frac{d}{d \theta} W^{2} d x d \theta+s \int_{D} \widetilde{\mathcal{A}} \frac{\partial \varphi}{\partial x_{j}} \frac{\partial}{\partial x_{j}}\left(\frac{d}{2 d \theta} W^{2}\right) d x d \theta
$$

and

$$
J_{2}=-s \int_{D} W^{2} \frac{d}{d \theta}(\widetilde{\mathcal{A}} \Delta \varphi) d x d \theta+s \int_{D} \widetilde{\mathcal{A}} \nabla \varphi \frac{d}{2 d \theta} \nabla W^{2} d x d \theta
$$

Then we have

$$
\begin{aligned}
I_{1}=\int_{\mathbb{T}^{2}} & {\left[\left(-s \frac{\partial \varphi}{\partial \theta}-s^{2} \widetilde{\mathcal{A}} \nabla|\varphi|^{2}\right) W^{2}\right]_{0}^{1} d x+s \int_{D} \frac{\partial^{2} \varphi}{\partial \theta^{2}} W^{2} d x d \theta+s^{2} \int_{D} \frac{\partial \widetilde{\mathcal{A}}}{\partial \theta}|\varphi|^{2} W^{2} d x d \theta } \\
& +s^{2} \int_{D} \widetilde{\mathcal{A}} \frac{\partial}{\partial \theta}|\varphi|^{2} W^{2} d x d \theta-s \int_{D} W^{2} \frac{d}{d \theta}(\widetilde{\mathcal{A}} \Delta \varphi) d x d \theta+s \int_{D} \widetilde{\mathcal{A}} \nabla \varphi \frac{d}{2 d \theta} \nabla W^{2} d x d \theta .
\end{aligned}
$$

For the second term, we have also

$$
\begin{aligned}
I_{2}=\left(P^{+} W, 2 s \widetilde{\mathcal{A}} \nabla \varphi \nabla W\right)_{L^{2}(D)}=\left(\left(-s \frac{\partial \varphi}{\partial \theta}-s^{2}|\nabla \varphi|^{2} \widetilde{\mathcal{A}}+s \nabla \cdot(\widetilde{\mathcal{A}} \nabla \varphi)\right) W, 2 s \widetilde{\mathcal{A}} \nabla \varphi \nabla W\right)_{L^{2}(D)} \\
=\left(\left(-s \frac{\partial \varphi}{\partial \theta}-s^{2}|\nabla \varphi|^{2} \widetilde{\mathcal{A}}\right) W, 2 s \widetilde{\mathcal{A}} \nabla \varphi \nabla W\right)_{L^{2}(D)}+(s \nabla \cdot(\widetilde{\mathcal{A}} \nabla \varphi) W, 2 s \widetilde{\mathcal{A}} \nabla \varphi \nabla W)_{L^{2}(D)} .
\end{aligned}
$$


The first scalar product can be written as follows

$$
J_{3}=-2 s \sum_{j} \int_{D}\left(s \frac{\partial \varphi}{\partial \theta}+s^{2}|\nabla \varphi|^{2} \widetilde{\mathcal{A}}\right) \widetilde{\mathcal{A}} \frac{\partial \varphi}{\partial x_{j}} W \frac{\partial W}{\partial x_{j}} d x d \theta
$$

giving

$$
J_{3}=s \sum_{j} \int_{D} \frac{\partial}{\partial x_{j}}\left[\left(s \frac{\partial \varphi}{\partial \theta}+s^{2}|\nabla \varphi|^{2} \widetilde{\mathcal{A}}\right) \widetilde{\mathcal{A}} \frac{\partial \varphi}{\partial x_{j}}\right] W^{2} d x d \theta .
$$

Deriving and regrouping the semblable term, we get

$$
\begin{gathered}
J_{3}=2 s \int_{D}\left[\nabla\left(s \frac{\partial \varphi}{\partial \theta}+s^{2}|\nabla \varphi|^{2} \widetilde{\mathcal{A}}\right) \widetilde{\mathcal{A}}+\left(s \frac{\partial \varphi}{\partial \theta}+s^{2}|\nabla \varphi|^{2} \nabla \widetilde{\mathcal{A}}\right)\right] \nabla \varphi W^{2} d x d \theta \\
+2 s^{2} \int_{D} \frac{\partial \varphi}{\partial \theta} \widetilde{\mathcal{A}} \Delta \varphi W^{2} d x d \theta+2 s^{3} \int_{D} \widetilde{\mathcal{A}}^{2}|\nabla \varphi|^{2} \Delta \varphi W^{2} d x d \theta
\end{gathered}
$$

The second term of $I_{2}$ satisfy the following

$$
J_{4}=2(\nabla \cdot(\widetilde{\mathcal{A}} \nabla W), 2 s \widetilde{\mathcal{A}} \nabla \varphi \nabla W)_{L^{2}(D)}=4 s \int_{D} \nabla \cdot(\widetilde{\mathcal{A}} \nabla W) \widetilde{\mathcal{A}} \nabla \varphi \nabla W d x d \theta
$$

Developing the expression $J_{4}$, we get

$$
J_{4}=4 s \int_{D}(\nabla \widetilde{\mathcal{A}} \nabla W+\widetilde{\mathcal{A}} \Delta W) \widetilde{\mathcal{A}} \nabla \varphi \nabla W d x d \theta=4 s \int_{D}\left(\widetilde{\mathcal{A}} \nabla \widetilde{\mathcal{A}} \nabla \varphi|\nabla W|^{2}+\widetilde{\mathcal{A}}^{2} \nabla \varphi \nabla W \Delta W\right) d x d \theta
$$

giving for $I_{2}$ the following equality

$$
\begin{array}{r}
I_{2}=2 s \int_{D}\left[\nabla\left(s \frac{\partial \varphi}{\partial \theta}+s^{2}|\nabla \varphi|^{2} \widetilde{\mathcal{A}}\right) \widetilde{\mathcal{A}}+\left(s \frac{\partial \varphi}{\partial \theta}+s^{2}|\nabla \varphi|^{2} \nabla \widetilde{\mathcal{A}}\right)\right] \nabla \varphi W^{2} d x d \theta+2 s^{2} \int_{D} \frac{\partial \varphi}{\partial \theta} \widetilde{\mathcal{A}} \Delta \varphi W^{2} d x d \theta \\
+2 s^{3} \int_{D} \widetilde{\mathcal{A}}^{2}|\nabla \varphi|^{2} \Delta \varphi W^{2} d x d \theta+4 s \int_{D}\left(\widetilde{\mathcal{A}} \nabla \widetilde{\mathcal{A}} \nabla \varphi|\nabla W|^{2}+\widetilde{\mathcal{A}}^{2} \nabla \varphi \nabla W \Delta W\right) d x d \theta
\end{array}
$$

For the integral $I_{3}$ we have

$$
\begin{array}{r}
I_{3}=\left(P^{+} W, s \nabla \cdot(\widetilde{\mathcal{A}} \nabla \varphi) W\right)_{L^{2}(D)}=\left(\left(-s \frac{\partial \varphi}{\partial \theta}-s^{2}|\nabla \varphi|^{2} \widetilde{\mathcal{A}}+s \nabla \cdot(\widetilde{\mathcal{A}} \nabla \varphi)\right) W, s \nabla \cdot(\widetilde{\mathcal{A}} \nabla \varphi) W\right)_{L^{2}(D)} \\
=s \int_{D}\left(-s \frac{\partial \varphi}{\partial \theta}-s^{2}|\nabla \varphi|^{2} \widetilde{\mathcal{A}}\right) \nabla \cdot(\widetilde{\mathcal{A}} \nabla \varphi) W^{2} d x d \theta+s^{2} \int_{D}|\nabla \cdot(\widetilde{\mathcal{A}} \nabla \varphi)|^{2} W^{2} d x d \theta .
\end{array}
$$

By combining the formulas (63), (70), (71), we get the desired result.

Lemma 3.2 Let $W$ be the solution of (37) and $P^{+}$and $P^{-}$defined by (52) and (53). Then we have the following estimates:

$$
2\left(P^{+} W, P^{-} W\right) \geq 4 s^{3} \int_{0}^{1} \int_{\mathbb{T}^{2}} \widetilde{\mathcal{A}}^{2}|\nabla \varphi|^{2} \Delta \varphi|W|^{2} d x d \theta+4 s \int_{0}^{1} \int_{\mathbb{T}^{2}} \widetilde{\mathcal{A}} \nabla \widetilde{\mathcal{A}} \cdot \nabla \varphi|\nabla W|^{2} d x d \theta
$$

proof The proof of this lemma follows from minimization of the each term $I_{1}, I_{2}$ and $I_{3}$.

We have also the following proposition

Proposition 3.2 Forall $s \geq s_{0}$ and forall $W \in L^{2}\left(\mathbb{R}, L^{2}\left(\mathbb{T}^{2}\right)\right)$ verifying $W(\theta, x)=e^{s \varphi(\theta, x)} U(\theta, x)$, where $U$ is solution to (5), we have the following estimate

$$
c \int_{0}^{1} \int_{D} \widetilde{\mathcal{A}}\left(s|\nabla U|^{2}+s^{3}|U|^{2}\right) d x d \theta \leq \int_{0}^{1} \int_{\mathbb{T}^{2}}\left|e^{s \varphi}(\nabla \cdot \widetilde{\mathcal{C}}+h(\theta, x))\right|^{2} d x d \theta .
$$

Proof It follows from (54) that

$$
\int_{0}^{1} \int_{\mathbb{T}^{2}}\left\|e^{s \varphi}(\nabla \cdot \widetilde{\mathcal{C}}+h(\theta, x))\right\|^{2} d x d \theta=\|P W\|^{2}
$$

As

$$
\|P W\|^{2}=\left\|\left(P^{+} W, P^{-} W\right)\right\|^{2}=\left\|P^{+} W\right\|^{2}+\left\|P^{-} W\right\|^{2}+2\left(P^{+} W, P^{-} W\right)_{L^{2}(D)} .
$$


we deduce that,

$$
2\left(P^{+} W, P^{-} W\right)_{L^{2}(D)} \leq \int_{0}^{1} \int_{\mathbb{T}^{2}}\left\|e^{s \varphi}(\nabla \cdot \widetilde{\mathcal{C}}+h(\theta, x))\right\|^{2} d x d \theta
$$

Thus, because Lemma 3.2,

$$
4 s^{3} \int_{0}^{1} \int_{\mathbb{T}^{2}} \widetilde{\mathcal{A}}^{2}|\nabla \varphi|^{2} \Delta \varphi|W|^{2} d x d \theta+4 s \int_{0}^{1} \int_{\mathbb{T}^{2}} \widetilde{\mathcal{A}} \nabla \widetilde{\mathcal{A}} \cdot \nabla \varphi|\nabla W|^{2} d x d \theta \leq \int_{0}^{1} \int_{\mathbb{T}^{2}}\left\|e^{s \varphi}(\nabla \cdot \widetilde{\mathcal{C}}+h(\theta, x))\right\|^{2} d x d \theta
$$

In consequence, for all $s \geq s_{0}$ and $\forall W \in \mathcal{L}^{\infty}\left(\mathbb{R}, L^{2}\left(\mathbb{T}^{2}\right)\right)$ given by $W(t, \theta, x)=e^{s \varphi(\theta, x)} U(t, \theta, w)$, we can rewrite inegality (74) in terms of $U$. This equality, called Carlemann inequality is given by

$$
4 s^{3} \int_{0}^{1} \int_{\mathbb{T}^{2}} \widetilde{\mathcal{A}}^{2}|\nabla \varphi|^{2} \Delta \varphi|U|^{2} d x d \theta+4 s \int_{0}^{1} \int_{\mathbb{T}^{2}} \widetilde{\mathcal{A}} \nabla \widetilde{\mathcal{A}} \cdot \nabla \varphi|\nabla U|^{2} d x d \theta \leq \int_{0}^{1} \int_{\mathbb{T}^{2}}\|(\nabla \cdot \widetilde{\mathcal{C}}+h(\theta, x))\|^{2} d x d \theta .
$$

\subsection{Estimates of $\left(P^{+} W, P^{-} W\right)$ and $\left\|P^{-} W\right\|^{2}$}

Lemma 3.3 Under assumptions, there exist constant such that, the term $\left(P^{+} W, P^{-} W\right)$ satisfy the following estimates

$$
\left\|\left(P^{+} W, P^{-} W\right)\right\| \leq \tilde{C}_{1} \int_{0}^{1} \int_{\mathbb{T}^{2}}|W|^{2} d x d \theta+\tilde{C}_{2} \int_{0}^{1} \int_{\mathbb{T}^{2}}|\nabla W|^{2} d x d \theta
$$

where $\tilde{C}_{1}$ and $\tilde{C}_{2}$ are two constant depending on $s, \tilde{\mathcal{A}}$ and $\varphi$.

Proof The proof is done in fews steps. We first estimates each term of the scalar product $\left(P^{+} W, P^{-} W\right)$.

Denoting by $I_{1}$ the following integrals, we have

$$
\begin{aligned}
& I_{1}=\left(-s \frac{\partial \varphi}{\partial \theta} W, \frac{\partial W}{\partial \theta}\right) \\
&\left|I_{1}\right|=\left|-s \int_{0}^{1} \int_{\mathbb{T}^{2}} \frac{\partial \varphi}{\partial \theta} W \frac{\partial W}{\partial \theta} d x d \tau\right| \\
& \leq s \int_{0}^{1} \int_{\mathbb{T}^{2}}\left|\frac{\partial \varphi}{\partial \theta} W \frac{\partial W}{\partial \theta}\right| d x d t \\
& \leq s \int_{\mathbb{T}^{2}} \int_{0}^{1}\left|\frac{\partial \varphi}{\partial \theta}\right|\left[\frac{d}{2 d \theta}|W|^{2}\right] d x \\
& \leq \frac{s}{2} \int_{\mathbb{T}^{2}} \int_{0}^{1}\left|\frac{\partial \varphi}{\partial \theta}\right||W|^{2} d x \\
& \leq \frac{s c}{2}\|W\|_{L^{2}\left(0, T, L^{2}\left(\mathbb{T}^{2}\right)\right)}^{2}
\end{aligned}
$$

Estimate of

$$
\begin{aligned}
I_{2} & =\left(-s \frac{\partial \varphi}{\partial \theta} W, 2 s \widetilde{\mathcal{A}} \nabla \varphi \nabla W\right) \\
\left|I_{2}\right| & \leq 2 s^{2} \int_{0}^{1} \int_{\mathbb{T}^{2}}\left|\frac{\partial \varphi}{\partial \theta} \nabla \varphi \widetilde{\mathcal{A}}\right||W \nabla W| d x d \theta \\
& \leq 2 s^{2} \int_{0}^{1} \int_{\mathbb{T}^{2}}|\varphi \nabla \varphi|\left|\frac{\partial \widetilde{\mathcal{A}}}{\partial \theta}\right||W \nabla W| d x d \theta \\
& \leq 2 s^{2} C_{1} \int_{0}^{1} \int_{\mathbb{T}^{2}} \frac{1}{2} \nabla|W|^{2} d \theta d x \\
& \leq s^{2} C_{1} \int_{0}^{1}|\nabla W|^{2}
\end{aligned}
$$

Estimate of $I_{3}$

$$
\begin{aligned}
I_{3}= & -s^{2} \int_{0}^{1} \int_{\mathbb{T}^{2}} \Delta \varphi \frac{\partial \varphi}{\partial t} \widetilde{\mathcal{A}} W^{2} d x d t \\
& =C s^{2} \int_{0}^{1} \int_{\mathbb{T}^{2}}|W|^{2} d x d t
\end{aligned}
$$

Estimate of $I_{4}$

$$
\begin{aligned}
\left|I_{4}\right| & =s^{2} \int_{0}^{1} \int_{\mathbb{T}^{2}}|\nabla \varphi|^{2} \widetilde{\mathcal{A}}\left|W \frac{\partial W}{\partial \theta}\right| d x d \theta \\
& \leq C_{4} s^{2} \int_{\mathbb{T}^{2}} \int_{0}^{1}\left|W \frac{\partial W}{\partial \theta}\right| d \theta d x \\
& \leq C_{4} s^{2} \int_{\mathbb{T}^{2}} \int_{0}^{1} \frac{d}{2 d \theta}|W|^{2} d \theta d x \leq 0
\end{aligned}
$$

Estimate of $I_{5}$

$$
\begin{gathered}
\left|I_{5}\right|=\left.s^{3} \int_{0}^{1} \int_{\mathbb{T}^{2}}|| \nabla \varphi\right|^{3}|\widetilde{A}|^{2}|| W \nabla W \mid d x d t \\
\leq 2 s^{3} \int_{\mathbb{T}^{2}} \int_{0}^{1}|\nabla \varphi|(|\nabla \varphi||\widetilde{\mathcal{A}}|)^{2}|W \nabla W| d t \\
\leq 2 C_{5} s^{3} \int_{\mathbb{T}^{2}} \int_{0}^{1} \nabla|W|^{2} d x d \theta
\end{gathered}
$$

Estimation of $I_{6}$

$$
\begin{aligned}
I_{6}= & \int_{0}^{1} \int_{\mathbb{T}^{2}}\left(-s^{2}|\nabla \varphi|^{2} \widetilde{A} W\right)(S \widetilde{A} \Delta \varphi W) d x d t \\
& \leq C_{6} s^{3} \int_{0}^{1} \int_{\mathbb{T}^{2}} W^{2} d x d t
\end{aligned}
$$


Estimate of $I_{7}$

$$
\begin{aligned}
I_{7}= & s \int_{0}^{1} \int_{\mathbb{T}^{2}} \Delta \varphi \widetilde{\mathcal{A}} W \frac{\partial W}{\partial t} d x d \theta \\
& \leq C_{7} s \int_{0}^{1} \int_{\mathbb{T}^{2}}\left|\frac{1}{2} \frac{\partial}{d \theta}\right| W^{2} \mid d x d t \\
& \leq 0
\end{aligned}
$$

Estimate of $I_{8}$

$$
\begin{gathered}
I_{8}=\left(-s \frac{\partial \varphi}{\partial \theta}-\widetilde{\mathcal{A}} s^{2}|\nabla \varphi|^{2} W, s \nabla \cdot(\widetilde{\mathcal{A}} \nabla \varphi) W\right) \\
\leq C_{8} \int_{D}|W|^{2} d x d \theta
\end{gathered}
$$

Estimate of $I_{9}$

$$
\begin{gathered}
I_{9}=(-2 s \nabla \cdot(\widetilde{\mathcal{A}} \nabla W), s \nabla \cdot(\widetilde{\mathcal{A}} \nabla \varphi) W) \\
\leq C_{9} \int_{D}|\nabla W|^{2} d x d \theta .
\end{gathered}
$$

\section{Conclusion}

In this work, we are interested in the null controlability of a degenerated and singularly perturbed partial differential equation. We therefore show the controlability at any times $\mathrm{T}$ by taking inspiration from an observability result. A carlmann estimate is also proven. It would therefore be interesting to look at the numerical aspects of these problems.

\section{References}

[1] G. Allaire, Homogenization and two-scale convergence, SIAM J. Math. Anal. 23 (1992), 1482-1518.

[2] P. Cannarsa, P.Martinez and J. Vancostenoble, Global Carleman Estimates for Degenerate Parabolic Operators with Applications, American Mathematical Society,Vol 329, Num. 1133, 2016.

[3] J. M. Coron and Guerrero S, Null Controllability of the $N$-Dimensional Stokes system with $N-1$ scolar controls, J. of Diff. Equ., 2009246 2908-21.

[4] J. M. Coron and S. Guerrero, Singular optimal control: a linear 1D parabolic-hyperbolic: example Ssymptotic Anal 44 237-57.
[5] J. M. Coron and S. Guerrero, Null Controllability of the $N$-Dimensional Stokes system with $N-1$ scolar controls, J. Diff. Equ. 2009, 246 2908-21.

[6] A. Dubova, A. Osses and J. P.Puel, Exact controllability to trajectoire for semi linear heat equations with discontinuous diffusion coefficient ESAIM: Control option calc, 2002 Var 8 621-61.

[7] A. Dubova, E. Fernandez-Cara, M. Gonzales-Burgos, Controlability results for discontinuous semilinear parabolic partial differenntial equations, C. R. Acad. Sci., t. 326, p. 1391-1395, 1998.

[8] A. Dubova, E. Fernandez-Cara, M. Gonzales-Burgos and E. Zuazua, On the controlability of parabolic systems with a nonlinear term involving the state and the gradient, SIAM J. Control. Optim, Vol. 41, N.3. p. 798-819, 2002.

[9] I. Faye, E. Frénod, D. Seck, Singularly perturbed degenerated parabolic equations and application to seabed morphodynamics in tided environment, Discrete and Continuous Dynamical Systems, Vol $29 N^{o} 3$ March 2011, pp 1001-1030.

[10] E. Frénod, Raviart P. A., and E. Sonnendrücker, Asymptotic expansion of the Vlasov equation in a large external magnetic field, J. Math. Pures et Appl. 80 (2001), 815-843.

[11] A. V. Fursikov, O. Y. Imanuvilov, Controlability of evolution equations, Suhak Kanguirok, Seoul National University, 1996.

[12] G. Lebeau, L. Robbiano, Contrôle exact de l'équation de la chaleur, Séminiare Equations aux dérivées partielles (dit"Goulaouic- Schwarttz), ppp 1-11, 94-95.

[13] J. L. Lions, Controlabilé exacte, perturbations et stabilisation de systèmes distribués; Perturbations, Recherche en Mathématiques Appliquées. Masson, 1998.

[14] K. Mauffrey, Contrôlabilité de systèmes gouvernés par des quations aux dérivées partielles, Analysis of PDEs [math.AP]. Université de Franche-Comté, 2012. French. $<$ tel-00864091v1>

[15] D. L. Russel, Controlability ans stability theory for linear partial differential equations: recent progress and open questions. Siam Review, 20 (4), pp 639-739, 1978.

[16] G. Nguetseng, A general convergence result for a functional related to the theory of homogenization, SIAM J. Math. Anal. 20 (1989), 608-623. 